\title{
Anti-fatigue activities of polysaccharides extracted from Hericium erinaceus
}

\author{
JIANQING LIU ${ }^{1}$, CONGXIN DU $^{1}$, YIFEI WANG ${ }^{1}$ and ZHIHUA YU ${ }^{2}$ \\ ${ }^{1}$ Section of Basketball, Wuhan Institute of Physical Education, Wuhan, Hubei 430079; \\ ${ }^{2}$ School of Physical Education, South-Central University for Nationalities, Hongshan, \\ Wuhan, Hubei 430074, P.R. China
}

Received April 16, 2014; Accepted December 5, 2014

DOI: $10.3892 /$ etm.2014.2139

\begin{abstract}
Hericium erinaceus (HEP) is a notable medicinal fungus grown in China and other oriental countries. Polysaccharides from HEP have recently attracted considerable attention due to their numerous physiological activities. The objective of this study was to evaluate the anti-fatigue activity of HEP in a mouse model. After one week of acclimation, mice were randomly divided into four groups: a control group, a low-dose HEP-treated group, a moderate-dose HEP-treated group, and a high-dose HEP-treated group. The treated groups received HEP (50, 100 and $200 \mathrm{mg} / \mathrm{kg}$, ig), while the control group received saline solution. Following treatment for 28 days, the mice performed a forced swimming test until they were exhausted, then the exhaustive swimming time was recorded along with certain biochemical parameters related to fatigue, including blood lactic acid (BLA), serum urea nitrogen (SUN), tissue glycogen, superoxide dismutase (SOD), glutathione peroxidase (GPx) and malondialdehyde (MDA). These results suggested that HEP has significant anti-fatigue activity by decreasing BLA, SUN and MDA content, as well as increasing tissue glycogen content and antioxidant enzyme activity. Based on these results, this study provided theoretical support for the application of HEP in the field of sports nutrition.
\end{abstract}

\section{Introduction}

Fatigue may be defined as a situation in which the capacity for work is diminished and efficiency of accomplishment reduced (1), and it can be classified as physical or mental,

Correspondence to: Professor Zhihua Yu, School of Physical Education, South-Central University for Nationalities, National Road 182, Hongshan, Wuhan 430074, P.R. China

E-mail: zhihuayu@126.com

Key words: forced swimming test, blood lactic acid, serum urea nitrogen, glycogen, superoxide dismutase, glutathione peroxidase, malondialdehyde, mice depending on its cause. As examples, physical fatigue is caused by excessive exercise and mental fatigue is caused by sleep deprivation (2). Physical fatigue may be accompanied by deterioration in performance. Several factors have been identified to contribute to physical fatigue. First, exercise promotes consumption of energy sources including glycogen by mobilizing the internal energy metabolism to the maximum and using and depleting the energy source (3). Second, exercise causes the production and accumulation of metabolic products including lactic acid and ammonia in the body (4). Third, intense exercise produces a large quantity of reactive oxygen species (ROS) due to increased oxygen consumption. The superoxide anion radical $\left(\mathrm{O}_{2}^{-}\right)$and hydrogen peroxide are generated as metabolic intermediates in the presence of oxygen. These may lead to a disturbance in the homeostasis of the endogenous antioxidative defense systems in the body, resulting in the development of fatigue (5). To date, pharmacological drugs or therapies used for treating fatigue have not been effective. Recently, interest has increased in the use of natural substance supplements for the attenuation of exercise-induced physical fatigue.

Hericium erinaceus (HEP) belongs to the Aphyllophorales, Hydnaecae and Hericium families and its fruiting body is called 'Houtou' in Chinese. It has been used as an edible and medicinal fungus in China and other oriental countries and areas for a number of years (6). The fungus contains essential constituents, including polysaccharides, lectins, proteins, lipids, hericenone, erinacol, erinacine and terpenoids (7). Polysaccharides from HEP have attracted considerable attention due to their numerous physiological activities, including immunomodulatory, hepatoprotective, antitumor, anti-aging, antioxidant and hypoglycemic activities (8-11). However, the anti-fatigue activity of HEP has not been investigated until now. The present study was designed to evaluate the anti-fatigue activity of HEP in a mouse model.

\section{Materials and methods}

Material. Dried fruiting bodies of HEP were obtained from a market in Wuhan city and identified by Professor Ming Wang from the Hubei Society for Microbiology (Wuhan, China). Voucher specimens (EH-SCN1391) were preserved in Hubei Natural Product Research Institute (Wuhan, China). 
Reagents and kits. Glucose was purchased from Guoyao Chemical Reagent Factory (Shanghai, China). The diagnostic kits for blood lactic acid (BLA), tissue glycogen and malondialdehyde (MDA) were purchased from Jiancheng Bioengineering Institute (Nanjing, China). The diagnostic kits for serum urea nitrogen (SUN) were purchased from Biosino Biotechnology and Science Inc. (Beijing, China). The diagnostic kits for superoxide dismutase (SOD) and glutathione peroxidase (GPx) were purchased from Comin Biotechnology Co., Ltd. (Suzhou, China). Other commercial chemicals used in the experiments were of analytical grade and were purchased from the Hongshan Reagent Company (Wuhan, China).

Preparation of polysaccharides from HEP. Polysaccharides from HEP were prepared using the ethanol precipitation method as described by Li et al (12) and modified by Hui et al (13). Briefly, dried fruits of HEP were ground and extracted with petroleum ether at $60^{\circ} \mathrm{C}$ for $4 \mathrm{~h}$ to remove colored materials, oligosaccharides and small molecule materials under reflux in the apparatus. The organic solvent was separated by centrifugation (4390 x g, $20 \mathrm{~min}$ ) and pretreated powder was obtained.

Next, the dried pretreated powder was extracted with boiling water (at a ratio of 1:30 w/v) for $4 \mathrm{~h}$. The mixture was centrifuged (4390 x g, $20 \mathrm{~min})$ and filtered, and the insoluble residue was treated again as mentioned above. The supernatant was incorporated and concentrated using a rotary evaporator at $50^{\circ} \mathrm{C}$ under a vacuum. The concentrated extract was precipitated by the addition of $95 \%(\mathrm{v} / \mathrm{v})$ ethanol to a final concentration of $80 \%(\mathrm{v} / \mathrm{v})$ and incubated for $12 \mathrm{~h}$ at $4^{\circ} \mathrm{C}$. The precipitate was collected by centrifugation (4390 x g, $20 \mathrm{~min}$ ) and then vacuum-dried at $40^{\circ} \mathrm{C}$ to afford crude polysaccharides from HEP. The polysaccharide content was measured by the phenol-sulfuric acid method using glucose as standard.

Experiment animals. Male ICR mice, weighing 18-20 g at the beginning of the study, were purchased from Wanqian Jiaxing Biotechnology Co, Ltd. (Wuhan, China). They were fed under controlled environmental conditions of temperature $\left(22 \pm 2^{\circ} \mathrm{C}\right)$ and a 12-h light/dark cycle, and maintained on a standard rodent diet and tap water ad libitum unless otherwise stated. All animals received professional humane care in compliance with the guidelines of the Ethical Committee of Wuhan Institute of Physical Education (Wuhan, China).

Experiment design. After one week of acclimation, the mice were randomly divided into four groups (ten mice in each group) as follows: i) control (C) group: the mice were allowed free access to a standard rodent diet and treated with saline solution; ii) low-dose HEP-treated (LHT) group: the mice were allowed free access to a standard rodent diet and treated with $50 \mathrm{mg} / \mathrm{kg}$ bw of HEP; iii) moderate-dose HEP-treated (MHT) group: the mice were allowed free access to a standard rodent diet and treated with $100 \mathrm{mg} / \mathrm{kg}$ bw of HEP; iv) High-dose HEP-treated (HHT) group: the mice were allowed free access to a standard rodent diet and treated with $200 \mathrm{mg} / \mathrm{kg}$ bw of HEP.

HEP was dissolved in $2.0 \mathrm{ml}$ saline solution, and the control group received the same volume of saline solution. Treatments were administered orally by gavage using a feeding needle, once a day for 28 consecutive days.

Forced swimming test. One hour after the final treatment, forced swimming tests (FSTs) were conducted using the method described by Zhang et al (14). Tests were carried out in an acrylic plastic pool $(90 \times 45 \times 45 \mathrm{~cm}) 35 \mathrm{~cm}$ deep with water maintained at $25 \pm 2^{\circ} \mathrm{C}$. A tin wire ( $5 \%$ of body weight) was loaded on the tail root of each mouse. Exhaustion was determined by observing loss of coordinated movements and failure to return to the surface within $10 \mathrm{sec}$, and the exhaustive swimming time was immediately recorded.

Analysis of biochemical parameters related to fatigue. After FSTs, the animals were sacrificed immediately by decapitation under anesthesia with sodium pentobarbital $(40 \mathrm{mg} / \mathrm{kg}$ bw, ip). Blood samples of the mice were respectively collected in heparinized tubes and tubes without anticoagulant. Blood plasma was prepared by centrifugation at $4^{\circ} \mathrm{C}(2919 \mathrm{x} \mathrm{g}$, $10 \mathrm{~min}$ ) for the BLA analysis, and serum was prepared by centrifugation at $4^{\circ} \mathrm{C}(2919 \mathrm{x} \mathrm{g}, 15 \mathrm{~min})$ for the SUN analysis. After the blood was collected, the gastrocnemius muscles and liver were rapidly excised and immediately frozen in liquid nitrogen and stored at $-80^{\circ} \mathrm{C}$ for the tissue glycogen, SOD, GPx and MDA analysis.

Analytical methods. The BLA content was determined based on the lactate dehydrogenase enzymatic method and the absorbance was read at $530 \mathrm{~nm}$ (15). SUN content was determined by the diacetyl monoxime colorimetric method and the absorbance was read at $520 \mathrm{~nm}$ (16). Glycogen content was determined by the sulfuric anthrone method and the absorbance was read at $620 \mathrm{~nm}$ (17). SOD activity was determined by the xanthine oxidase method (hydroxylamine method) and the absorbance was read at $550 \mathrm{~nm}$ (18). GPx activity was determined by the dithio-binitrobenzoic acid method and the absorbance was read at $412 \mathrm{~nm}$ (19). MDA content was determined by the thiobarbituric acid method and the absorbance was read at $532 \mathrm{~nm}(20)$.

Statistical analysis. The results are expressed as the means \pm standard deviation. Comparisons between groups were made using Student's t-test, and $\mathrm{P}<0.05$ was considered to indicate a statistically significant difference.

\section{Results and Discussion}

Effects of HEP on exhaustive swimming times. The FST, a behavioral test for rodents, previously used to predict the efficacy of antidepressants, has recently been used to examine whether certain agents have anti-fatigue activities (21). Prolonged swimming times in an FST indicate a decrease in fatigue (22).

The effects of HEP on exhaustive swimming times are shown in Fig. 1. Exhaustive swimming times in the LHT, MHT and HHT groups were significantly longer $(\mathrm{P}<0.05)$ than that in the $\mathrm{C}$ group, by $18.15,37.18$ and $58.46 \%$, respectively. These results indicated that HEP had significant anti-fatigue activity and was capable of elevating the exercise tolerance in mice. 


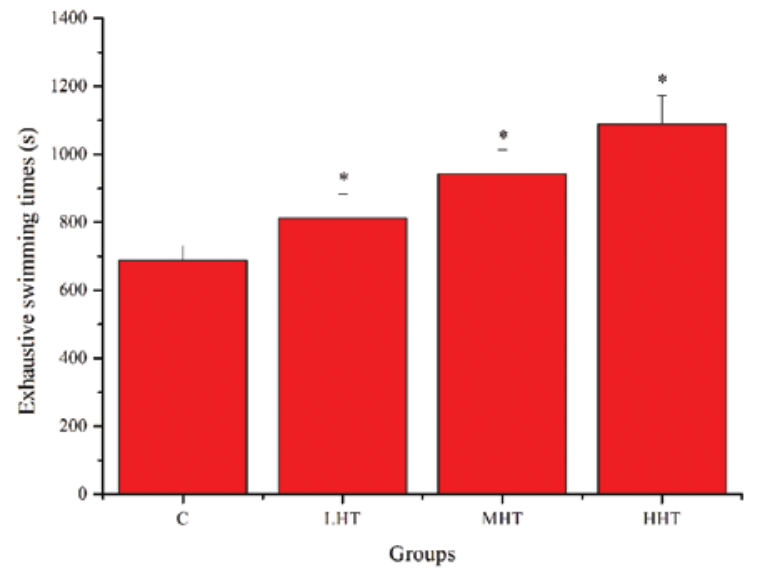

Figure 1. Effects of Hericium erinaceus on exhaustive swimming times Values are expressed as the means $\pm \mathrm{SD}$. ${ }^{*} \mathrm{P}<0.05$, compared with the C group. C, control; LHT, low-dose HEP-treated group; MHT, moderate-dose HEP-treated group; HHT, high-dose HEP-treated group.

Effects of HEP on BLA and SUN content. In general, the swimming exercise is known to induce blood biochemical changes (23). The muscle produces a considerable amount of lactic acid when it obtains sufficient energy from anaerobic glycolysis, and the increased concentration of lactic acid brings about a reduction in the $\mathrm{pH}$ of muscle tissue and blood, which could induce various biochemical and physiological side effects, including glycolysis and phosphofructokinase and calciumion release, through muscular contraction (24). Therefore, BLA is a sensitive index of fatigue status. Urea is formed in the liver as the end product of protein metabolism. During digestion, protein is broken down into amino acids. Amino acids contain nitrogen, which is removed as $\mathrm{NH}_{4}^{+}$ (an ammonium ion), while the remainder of the molecule is used to produce energy and other substances required by the cell (14). There is a positive correlation between the urea nitrogen in vivo and exercise tolerance. In other words, the worse the body is adapted for exercise tolerance, the more significantly the urea nitrogen level increases (25). Thus, SUN is another sensitive index of fatigue status.

The effects of HEP on BLA and SUN content are shown in Fig. 2. The BLA content of the LHT, MHT and HHT groups was significantly lower $(\mathrm{P}<0.05)$ than that of the $\mathrm{C}$ group, by $16.43,28.90$ and $52.13 \%$, respectively. The SUN content of the MHT and HHT groups was significantly lower $(\mathrm{P}<0.05)$ than that of the C group, by 20.87 and $27.04 \%$, respectively. The SUN content of the LHT group was also lower, but not significantly $(\mathrm{P}>0.05)$. These results indicated that HEP effectively delayed the increase in BLA, reduced the catabolism of protein for energy and increased the adaptive capacity to exercise load, which ultimately postponed the appearance of physical fatigue.

Effects of HEP on glycogen content in liver and muscle. Energy for exercise is derived initially from the breakdown of glycogen in muscle. Following strenuous exercise, it may be depleted, and at later stages the energy will be derived from hepatic glycogen (26). Therefore, the depletion of glycogen stores may be a significant factor in the development of fatigue.

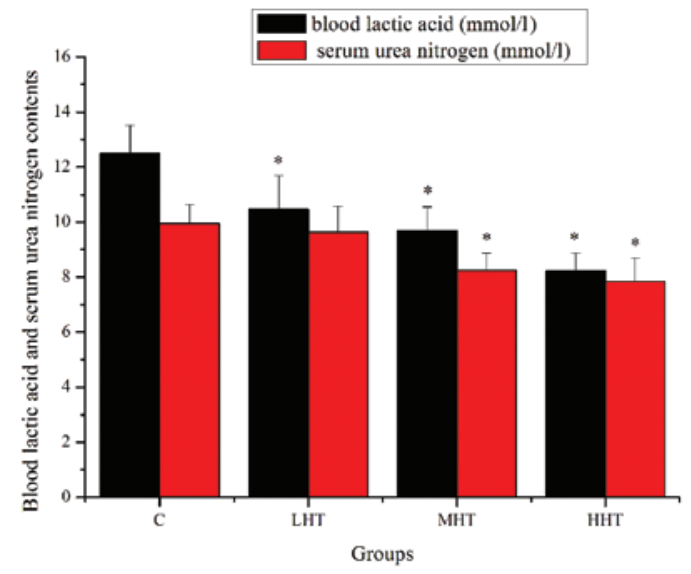

Figure 2. Effects of Hericium erinaceus on blood lactic acid and serum urea nitrogen content. Values are expressed as the means \pm SD. ${ }^{*} \mathrm{P}<0.05$, compared with the C group. C, control; LHT, low-dose HEP-treated group; MHT, moderate-dose HEP-treated group; HHT, high-dose HEP-treated group.

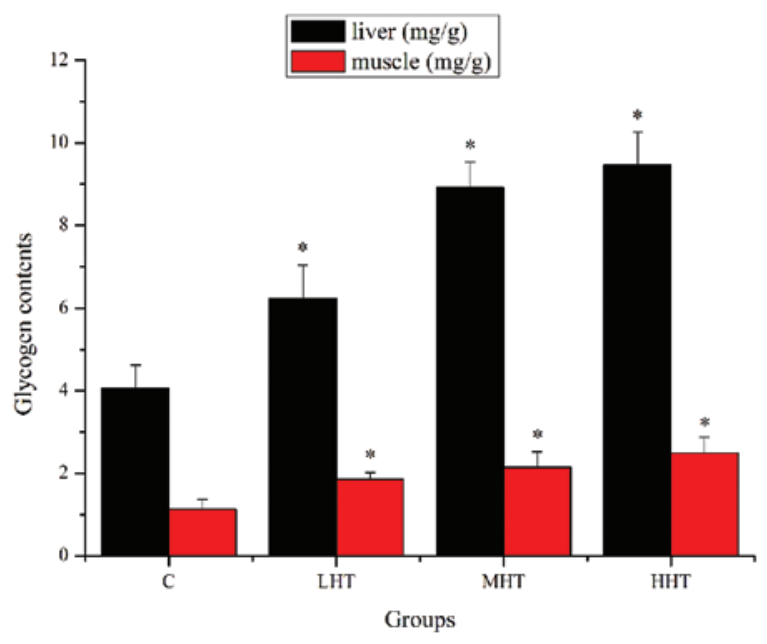

Figure 3. Effects of Hericium erinaceus on glycogen content in liver and muscle. Values are expressed as the means $\pm \mathrm{SD}$. ${ }^{*} \mathrm{P}<0.05$, compared with the C group. C, control; LHT, low-dose HEP-treated group; MHT, moderate-dose HEP-treated group; HHT, high-dose HEP-treated group.

The effects of HEP on glycogen content in liver and muscle are shown in Fig. 3. The liver glycogen content of the LHT, MHT and HHT groups was significantly higher $(\mathrm{P}<0.05)$ than that of the $\mathrm{C}$ group, by 53.45, 119.70 and $133.25 \%$, respectively. The muscle glycogen content of the LHT, MHT and HHT groups was significantly higher $(\mathrm{P}<0.05)$ than that of the $\mathrm{C}$ group, by $64.60,90.27$ and $120.35 \%$, respectively. These results indicated that HEP may contribute to the improvement of metabolic control of exercise and the activation of energy metabolism (27), which could ameliorate physical fatigue by increasing the storage of glycogen in liver and muscle.

Effects of HEP on SOD and GPX activity in liver and muscle. Previous studies have reported that ROS are responsible for exercise-induced protein oxidation, and contribute significantly to muscle fatigue (28). Two major classes of endogenous 


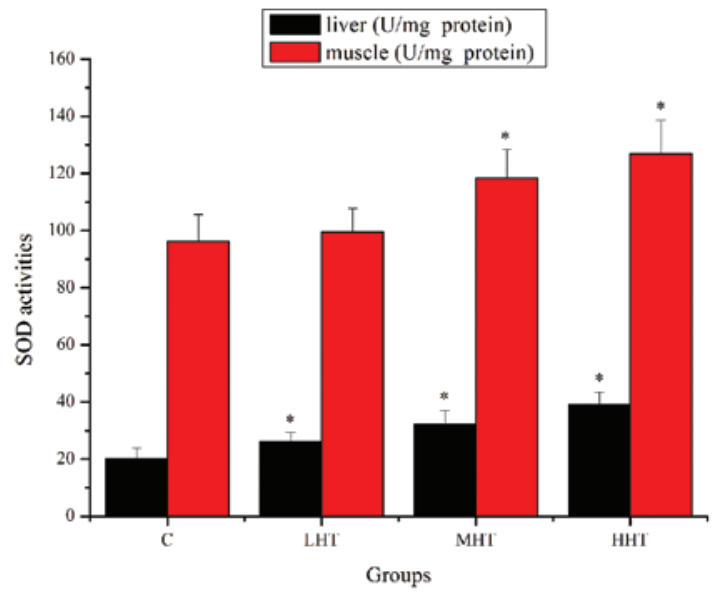

Figure 4. Effects of Hericium erinaceus on superoxide dismutase (SOD) activity in liver and muscle. Values are expressed as the means $\pm \mathrm{SD} .{ }^{*} \mathrm{P}<0.05$, compared with the C group. C, control; LHT, low-dose HEP-treated group; MHT, moderate-dose HEP-treated group; HHT, high-dose HEP-treated group.

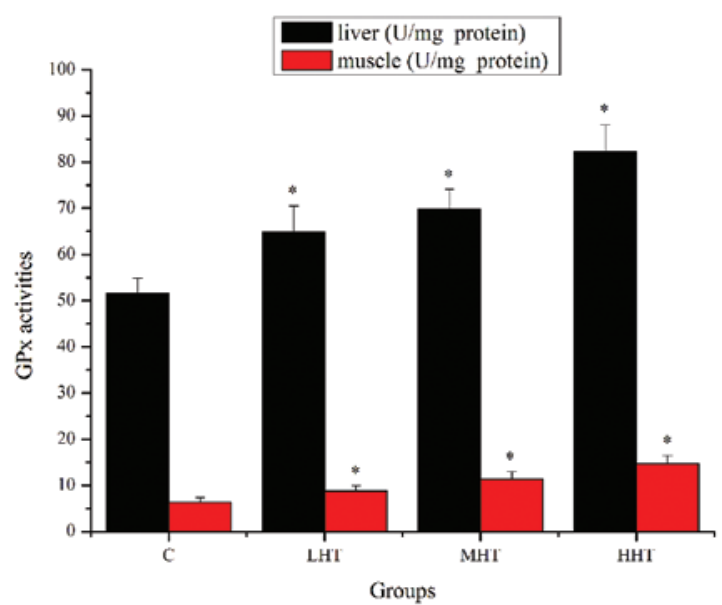

Figure 5. Effects of Hericium erinaceus on glutathione peroxidase (GPx) activity in liver and muscle. Values are expressed as the means \pm SD ${ }^{*} \mathrm{P}<0.05$, compared with the $\mathrm{C}$ group. C, control; LHT, low-dose HEP-treated group; MHT, moderate-dose HEP-treated group; HHT, high-dose HEP-treated group.

protective mechanisms, enzymatic and non-enzymatic antioxidants, work to reduce the harmful effects of ROS in cells (29). SOD and GPx constitute the principal components of the enzymatic antioxidant defense systems. There is growing evidence indicating that the improvement in the activity of SOD and GPx help fight against fatigue and protect cells from oxidative damage $(30,31)$.

The effects of HEP on SOD activity are shown in Fig. 4. The SOD activity in the liver of the LHT, MHT and HHT groups was significantly higher $(\mathrm{P}<0.05)$ than in that of the C group, by $29.96,60.66$ and $94.39 \%$, respectively. SOD activity in the muscle of the MHT and HHT group was significantly higher $(\mathrm{P}<0.05)$ than in that of the $\mathrm{C}$ group, by 22.96 and $31.81 \%$, respectively. SOD activity in the muscle of the LHT group was also higher but not significantly $(\mathrm{P}>0.05)$. The effects of HEP on GPx activity are shown in Fig. 5. GPx

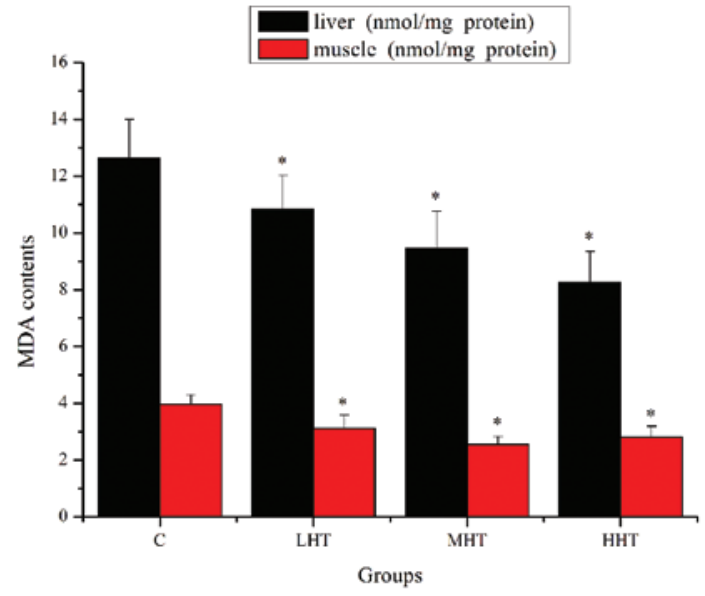

Figure 6. Effects of Hericium erinaceus on malondialdehyde (MDA) content in muscle and liver. Values are expressed as means \pm SD. ${ }^{*} \mathrm{P}<0.05$, compared with the C group. C, control; LHT, low-dose HEP-treated group; MHT, moderate-dose HEP-treated group; HHT, high-dose HEP-treated group.

activity in the liver of the LHT, MHT and HHT groups was significantly higher $(\mathrm{P}<0.05)$ than in that of the $\mathrm{C}$ group, by $25.50,35.11$ and $59.39 \%$, respectively. GPx activity in the muscle of the LHT, MHT and HHT groups was significantly higher $(\mathrm{P}<0.05)$ than in that of the $\mathrm{C}$ group, by $37.74,78.77$ and $130.35 \%$, respectively. These results indicated that HEP was able to upregulate antioxidant enzymes activity to ameliorate physical fatigue.

Effects of HEP on MDA content in muscle and liver. It is generally accepted that fatigue causes the release of ROS, which leads to lipid peroxidation of the membrane structure and causes oxidative damage to cellular macromolecules (32). MDA is the breakdown product of the major chain reactions leading to the oxidation of polyunsaturated fatty acids and thus serves as an indicator of lipid peroxidation (33). A number of studies have reported that exhaustive exercise increased the MDA content in liver and muscle tissues in rats and mice $(34,35)$.

The effects of HEP on MDA content are shown in Fig. 6. The MDA content in the liver of the LHT, MHT and HHT groups was significantly lower $(\mathrm{P}<0.05)$ than in that of the C group, by $16.71,33.62$ and $52.84 \%$, respectively. The MDA content in the muscle of the LHT, MHT and HHT groups was significantly lower $(\mathrm{P}<0.05)$ than that of the $\mathrm{C}$ group, by $26.92,54.69$ and $40.93 \%$, respectively. These results indicated that HEP reduced lipid peroxidation and prevented exercise-induced oxidative damage.

The results of the present study suggest that HEP possesses significant anti-fatigue activity by decreasing BLA, SUN and MDA content, and increasing tissue glycogen content and antioxidant enzyme activity. Based on these results, this study provides theoretical support for the application of HEP in the field of sports nutrition.

\section{Acknowledgements}

This study was supported by the Department of Education of Hubei Province, China (grant no. 20120484). 


\section{References}

1. Zhang G,Zhou SM, Tian JH, Huang QY and Gao YQ: Anti-fatigue effects of methazolamide in high-altitude hypoxic mice. Trop J Pharm Res 11: 209-215, 2012.

2. Yan F, Zhang Y and Wang BB: Effects of polysaccharides from Cordyceps sinensis mycelium on physical fatigue in mice. Bangladesh J Pharmacol 7: 217-221, 2012.

3. Wang L, Zhang HL, Lu R, Zhou YJ, Ma R, Lv JQ, Li XL, Chen LJ and Yao Z: The decapeptide CMS001 enhances swimming endurance in mice. Peptides 29: 1176-1182, 2008.

4. Ikeuchi M, Koyama T, Takei S, Kino T and Yazawa K: Effects of Benzylglucosinolate on endurance capacity in mice. J Health Sci 55: 178-182, 2009

5. Blokhina O, Virolainen E and Fagerstedt KV: Antioxidants, oxidative damage and oxygen deprivation stress: a review. Ann Bot 91 Spec No: 179-184, 2003.

6. Lee JS, Min KM, Cho JY and Hong EK: Study of macrophage activation and structural characteristics of purified polysaccharides from the fruiting body of Hericium erinaceus. J Microbiol Biotechnol 19: 951-959, 2009.

7. Zhu Y, Li Q, Mao G, Zou Y, Feng W, Zheng D, Wang W, Zhou L, Zhang T, Yang J, Yang L and Wu X: Optimization of enzyme-assisted extraction and characterization of polysaccharides from Hericium erinaceus. Carbohydr Polym 101: 606-613, 2014

8. Zhang Z, Lv G, Pan H, Pandey A, He W and Fan L: Antioxidant and hepatoprotective potential of endo-polysaccharides from Hericium erinaceus grown on tofu whey. Int J Biol Macromol 51 1140-1146, 2012.

9. Wang JC, $\mathrm{Hu} \mathrm{SH}, \mathrm{Su} \mathrm{CH}$ and Lee TM: Antitumor and immunoenhancing activities of polysaccharide from culture broth of Hericium spp. Kaohsiung J Med Sci 17: 461-467, 2001.

10. Khan MA, Tania M, Liu R and Rahman MM: Hericium erinaceus: an edible mushroom with medicinal values. J Complement Integr Med 10: 1-6, 2013

11. Han ZH, Ye JM and Wang GF: Evaluation of in vivo antioxidant activity of Hericium erinaceus polysaccharides. Int $\mathrm{J}$ Biol Macromol 52: 66-71, 2013.

12. Li FL, Li QW, Gao DW, Peng Y and Feng CN: Preparation and antidiabetic activity of polysaccharide from Portulaca oleracea $\mathrm{L}$. Afr J Biotechnol 8: 569-573, 2009.

13. Hui MK, Wu WK, Shin VY, So WH and Cho CH: Polysaccharides from the root of Angelica sinensis protect bone marrow and gastrointestinal tissues against the cytotoxicity of cyclophosphamide in mice. Int J Med Sci 3: 1-6, 2006.

14. Zhang XL, Ren F, Huang W, Ding RT, Zhou QS and Liu XW: Anti-fatigue activity of extracts of stem bark from Acanthopanax senticosus. Molecules 16: 28-37, 2010.

15. Shang Y, Cheng J, Qi J and Miao H: Scutellaria flavonoid reduced memory dysfunction and neuronal injury caused by permanent global ischemia in rat. Pharmacol Biochem Behav 82: 67-73, 2005.

16. Wybenga DR, Di Giorgio J and Pileggi VJ: Manual and automated methods for urea nitrogen measurement in whole serum. Clin Chem 17: 891-895, 1971.

17. Sotelo-Félix JI, Martinez-Fong D, Muriel P, Santillán RL, Castillo D and Yahuaca P: Evaluation of the effectiveness of Rosmarinus officinalis (Lamiaceae) in the alleviation of carbon tetrachloride-induced acute hepatotoxicity in the rat. J Ethnopharmacol 81: 145-154, 2002.
18. Huang H, Shan J, Pan XH, Wang HP and Qian LB: Carvedilol protected diabetic rat hearts via reducing oxidative stress. J Zhejiang Univ Sci B 7: 725-731, 2006.

19. Zhang QH, Wu CF, Yang JY, Mu YH, Chen XX and Zhao YQ Reduction of cyclophosphamide-induced DNA damage and apoptosis effects of ginsenoside $\mathrm{Rb}$ (1) on mouse bone marrow cells and peripheral blood leukocytes. Environ Toxicol Pharmacol 27: 384-389, 2009.

20. Chen H, Sun YP, Li Y, Liu WW, Xiang HG, Fan LY, Sun Q, Xu XY, Cai JM, Ruan CP, et al: Hydrogen-rich saline ameliorates the severity of 1-arginine-induced acute pancreatitis in rats. Biochem Biophys Res Commun 393: 308-313, 2010.

21. Koo HN, Lee JK, Hong SH and Kim HM: Herbkines increases physical stamina in mice. Biol Pharm Bull 27: 117-119, 2004.

22. Tan W, Yu KQ, Liu YY, Ouyang MZ, Yan MH, Luo R and Zhao XS: Anti-fatigue activity of polysaccharides extract from Radix Rehmanniae Preparata. Int J Biol Macromol 50: 59-62, 2012.

23. An HJ, Choi HM, Park HS, Han JG, Lee EH, Park YS, Um JY, Hong SH and Kim HM: Oral administration of hot water extracts of Chlorella vulgaris increases physical stamina in mice. Ann Nutr Metab 50: 380-386, 2006.

24. Huang CC, Hsu MC, Huang WC, Yang HR and Hou CC: Triterpenoid-rich extract from antrodia camphorata improves physical fatigue and exercise performance in mice. Evid Based Complement Alternat Med 2012: 364741, 2012.

25. Tang W, Zhang Y, Gao J, Ding X and Gao S: The anti-fatigue effect of $20(\mathrm{R})$-ginsenoside Rg3 in mice by intranasally administration. Biol Pharm Bull 31: 2024-2027, 2008.

26. Anand T, Phani Kumar G, Pandareesh MD, Swamy MS, Khanum F and Bawa AS: Effect of bacoside extract from Bacopa monniera on physical fatigue induced by forced swimming. Phytother Res 26: 587-593, 2012.

27. Xu C, Lv J, Lo YM, Cui SW, Hu X and Fan M: Effects of oat $\beta$-glucan on endurance exercise and its anti-fatigue properties in trained rats. Carbohydr Polym 26: 1159-1165, 2013.

28. Powers SK and Jackson MJ: Exercise-induced oxidative stress: cellular mechanisms and impact on muscle force production. Physiol Rev 88: 1243-1276, 2008.

29. Powers SK, DeRuisseau KC, Quindry J and Hamilton KL: Dietary antioxidants and exercise. J Sports Sci 22: 81-94, 2004.

30. Chen Z, Li S, Wang X and Zhang CL: Protective effects of Radix Pseudostellariae polysaccharides against exercise-induced oxidative stress in male rats. Exp Ther Med 5: 1089-1092, 2013.

31. Yan F, Wang B and Zhang Y: Polysaccharides from Cordyceps sinensis mycelium ameliorate exhaustive swimming exercise-induced oxidative stress. Pharm Biol 52: 157-161, 2014.

32. Prasad VMP and Khanum F: Antifatigue activity of ethanolic extract of Ocimum sanctum in rats. Res J Med Plant 6: 37-46, 2012.

33. Zhonghui Z, Xiaowei Z and Fang F: Ganoderma lucidum polysaccharides supplementation attenuates exercise-induced oxidative stress in skeletal muscle of mice. Saudi J Biol Sci 21: 119-123, 2014.

34. Chen QP and Wei P: Icariin supplementation protects mice from exercise-induced oxidant stress in liver. Food Sci Biotech 22: $1405-1413,2013$

35. Shan X, Zhou J, Ma T and Chai Q: Lycium barbarum polysaccharides reduce exercise-induced oxidative stress. Int J Mol Sci 12: 1081-1088, 2011. 